\title{
What keeps nurses from the sexual counseling of patients with heart failure?
}

\author{
Tialda Hoekstra, Ivonne Lesman-Leegte, Margriet F Couperus, \\ Robbert Sanderman and Tiny Jaarsma
}

\section{Linköping University Post Print}

N.B.: When citing this work, cite the original article.

Original Publication:

Tialda Hoekstra, Ivonne Lesman-Leegte, Margriet F Couperus, Robbert Sanderman and Tiny Jaarsma, What keeps nurses from the sexual counseling of patients with heart failure?, 2012, Heart \&amp; Lung, (41), 5, 492-499.

http://dx.doi.org/10.1016/j.hrtlng.2012.04.009

Copyright: Elsevier http://www.elsevier.com/

Postprint available at: Linköping University Electronic Press

http://urn.kb.se/resolve?urn=urn:nbn:se:liu:diva-84902 
What keeps nurses from sexual counseling of heart failure patients? 


\section{Abstract}

Objectives

To examine the current practice of discussing sexual health by heart failure (HF) nurses, and to explore which barriers prevent nurses from discussing sexuality. Methods

The Nurses' Survey of Sexual Counseling of MI patients' and a list of barriers were used to form a questionnaire, which was sent to all HF clinics $(n=122)$ in the Netherlands.

Results

The majority $(75 \%)$ of nurses $(n=146)$ felt a certain responsibility to discuss patients' sexual health. However, in practice $61 \%$ of the nurses rarely or never addressed sexuality. Barriers that prevented nurses from addressing sexuality and differed between nurses who do $(n=58)$ and do not $(n=88)$ discuss sexuality include lack of organizational policy ( $49 \%$ vs. $79 \%, p<.001)$ and lack of training (43\% vs. $80 \%$, $p<.001)$, and not knowing how to initiate the subject ( $24 \%$ vs. $72 \%, p<.001)$. Nurses preferred to address sexuality during a follow-up visit or when discussing medication. Conclusions

Although HF nurses feel responsible for discussing sexuality, this topic is rarely addressed in clinics. Several barriers were identified, relating to personal, patient and organizational factors.

Keywords: Heart failure; sexual health; sexual counseling; nurses' attitudes; barriers 
Abbreviations list

HF Heart failure

MI Myocardial infarction 


\section{Introduction}

Sexual problems are common amongst both male and female heart failure (HF) patients. ${ }^{1,2}$ Problems HF patients have reported include a decrease in sexual interest, a decrease in the frequency of sexual activity, severe negative changes in sexual performance, and loss of pleasure or satisfaction related to sexual activity. Furthermore, a significant proportion of men with HF experience erectile dysfunction. ${ }^{1-3}$ Patients attribute their sexual problems to their HF. In addition, HF medication may also lead to sexual problems. The use of beta-blockers in particular is known to have a possible detrimental effect on sexuality, although studies addressing the relationship between beta-blocker and sexual problems are incongruent. ${ }^{4,5}$

HF patients and their partners have expressed a wish to receive information about resuming sexual activity after being diagnosed with $\mathrm{HF}^{6}$ However, when trying to address this topic, patients experience barriers, such as the perception that individual practitioners do not appear to be experienced enough to understand the patient's problems, or because feelings of shyness and embarrassment prevent them from initiating discussions. ${ }^{7}$ In this respect nurses have an important role to play in assessing the effects of HF on sexuality with both patients and their partners. This becomes even more apparent when taking into account the fact that HF nurses are becoming more and more involved in the long-term management of HF patients.

Although international HF guidelines recommend that healthcare providers discuss sexual health with their patients, previous studies have shown that even though healthcare providers do feel responsible for discussing sexual health, they nevertheless rarely discuss this topic with their patients in practice..$^{8-11}$ To improve patient care in this area, it is important to learn which barriers prevent nurses from discussing sexual health 
with their patients. A study among cardiac rehabilitation staff showed that barriers experienced included lack of training, the feeling that the patient is not yet ready to discuss issues related to sexual health, and a general lack of knowledge of sexual health. $^{11}$

Unfortunately, there is little information available on the current practice of discussing sexual health and providing sexual counseling by HF nurses in the Netherlands. Therefore, the purpose of this study was to examine: (1) the current practice of discussing sexual health in HF clinics in the Netherlands and the perceived responsibility of nurses to address patients' sexual health, (2) the preferred moment during HF treatment to address patients' sexual concerns, and (3) differences in barriers to sexual counseling between nurses who do and who do not discuss this topic.

\section{Methods}

\section{Study participants}

A letter of introduction was sent to all HF clinics in the Netherlands $(n=122)$, which included three copies of a questionnaire and a freepost return envelope. ${ }^{12,13}$ We chose to include three copies, because in general a maximum of three HF nurses work at each HF clinic. In the letter of introduction nurses working at the specific HF clinic were asked to complete the (anonymous) questionnaire and to return it within 2 weeks. Non-responders received a reminder in the form of a telephone call after 3 weeks. Confidentiality was guaranteed to the participants of the study and answers could not be traced back to individual nurses.

\section{Questionnaire}


Two questionnaires used in previous studies were utilized to form the basis of the current questionnaire. These included two subscales of the Nurses Survey of Sexual Counseling of MI Patients and a list of barriers from the Irish CHARMS study $11,14,15$

1. The subscales practice and responsibility of the Nurses Survey of Sexual Counseling of Ml patients were used in this study:

"Practice" (8 items): This subscale addresses the overall practice of sexual counseling. For example, "In the past year, with what percentage of your clients have you assessed their sexual health?" Four items were scored on a scale of 0 to 3 , and 4 items from 0 to 5 . The subscale score ranges from 0 to 32 , with higher scores indicating better performance of nurses on sexual counseling in daily practice. To clarify the data, the scores on the 6 answer categories were translated into "never" (0\%), "sometimes" (1-20\% and 21-40\%), and "often" (41-60\%, 61-80\%, and $81-$ $100 \%)$.

'Responsibility' (5 items): This subscale addresses the perceived responsibility of nurses to provide sexual counseling. For example, "Nurses have a responsibility to discuss sexual health with their patients" ("strongly agree" to "strongly disagree"). All items could be scored on a scale from 0 to 4 . The subscale score ranges from 0 to 20 , with higher scores indicating a greater sense of responsibility to provide sexual counseling. Reliability of the subscales was assessed using Cronbach's alpha and ranged from 0.89 (practice) to 0.75 (responsibility). ${ }^{14}$

2. Barriers: The list of barriers that prevent nurses from discussing sexual health consisted of 16 items, which were created by Doherty et al. ${ }^{11}$ To gain more insight in 
other possible barriers, 8 additional items on barriers retrieved from discussions by a group of experts (including a cardiologist, a sexologist, HF nurses, and psychologists) were added to the list. These included: lack of organizational policy (e.g., is a protocol on sexual health available?), lack of knowledge on how to initiate the subject, the patient does not initiate the subject, sexuality is a private matter, feeling no connection with the patient, feeling uncomfortable addressing the topic, projecting personal feelings on the patient (e.g., "I would find it unpleasant myself"), and the patient is of the same sex. Scores on each item could range from 1 ("strongly disagree") to 5 ("strongly agree").

In addition, participants had to answer 10 questions on socio-demographics and on their professional background. Face validity of the total questionnaire was tested in a pilot study by $4 \mathrm{HF}$ nurses. Only editorial changes were made in response to the nurses' answers.

\section{Statistical analyses}

Data were analyzed using SPSS version 16.0 (SPSS Inc, Chicago, IL). Descriptive analyses were employed to test the sample and the responses to the study variables. $\mathrm{Chi}^{2}$-tests were used to compare the perceived barriers of nurses who do discuss sexual health with the barriers of nurses who do not discuss sexuality with their patients. A $p$ value of $<0.05$ was considered statistically significant. For comparing the barriers of nurses who do and who do not discuss sexual health, the scores on the barriers were recoded to agree ("strongly agree" and "agree") and disagree ("strongly disagree" and "disagree"). The answer category "somewhat (dis)agree" was not included in the analyses. Scores on discussing sexual health 
were based on answers to the first question of the practice subscale: "How often is sexual health assessed in your practice?" The scores were recoded into do discuss ("often" and "occasionally") and do not discuss ("rarely" and "never").

\section{Results}

\section{Study population}

In total, nurses from 88 of the 122 (72\%) HF clinics responded by returning at least one completed questionnaire. The final sample of respondents consisted of 146 nurses. The majority of nurses was female (83\%), with a mean age of $45( \pm 8)$ years (age range $25-61$ years). In total, $67 \%$ of nurses had a Bachelor degree and $30 \%$ reported to have gone on to higher education or followed workshops on sexuality in nursing practice (Table 1).

\section{Practice and responsibility}

The subscale "Practice" had a mean score of $10 \pm 4$ (range 2-24, higher scores indicate a better performance of nurses on discussing sexual health in daily practice). When looking at the specific items, $1 \%$ of nurses reported to frequently assess sexual health with their patients, $38 \%$ did so occasionally, $53 \%$ rarely, and $8 \%$ never assessed sexual health with their patients. Furthermore, $50 \%$ of nurses never referred patients with questions about sexuality to other professions (Figure 1), while $49 \%$ did so sometimes.

The subscale "Responsibility" had a mean score of $15 \pm 2$ (range 7-20, higher scores indicate a greater sense of responsibility of nurses to initiate sexual counseling). In total, $75 \%$ of nurses agreed that they have a responsibility to discuss sexual health with their clients; whereas $11 \%$ disagreed, and $14 \%$ were not sure. 
Moreover, $37 \%$ reported that it is usually appropriate to initiate a discussion on sexual health with patients, and $5 \%$ of nurses stated that it is always appropriate to initiate such a discussion (Figure 2).

No significant differences in background variables were found between nurses who do and nurses who do not discuss sexual health with their patients. However, significantly more nurses who do discuss sexual health had gone on to education on sexual counseling compared to nurses who do not discuss sexual health ( $41 \%$ vs. $22 \%, p=0.012)$ (Table 1).

\section{Preferred moment of discussing sexual health}

The majority of nurses (74\%) agreed that sexual health should be assessed during a follow-up visit at the HF clinic or when discussing medication with the patient (72\%). Thirty percent of nurses felt that sexual health should be discussed during the first visit to the HF clinic, 34\% preferred to discuss sexual health during cardiac rehabilitation, and $13 \%$ rather addressed this topic during hospital admission (Figure $3)$.

\section{Barriers to discussing sexual health}

The most prominent barriers that deter nurses from discussing sexual health with their patients included lack of organizational policy (67\%), lack of training (42\%), issues relating to culture and religion (40\%), and the age of the patient (39\%). Barriers mentioned in the questionnaire which did not keep nurses from discussing sexual health were that they do not perceive counseling to be their task (3\%), that the patient is of the same sex $(0 \%)$, or that the patient is of the opposite sex $(1 \%)$. 
We looked in more detail at the differences in barriers between nurses who report they do discuss ("often" and "occasionally") sexual health with their patients $(n=58)$ and those who do not discuss' ("rarely" and "never") this topic $(n=88)$.

Nurses who reported they do discuss sexual health with their patients mentioned the following barriers to addressing this topic: issues related to culture and religion (68\%), issues related to language and ethnicity (56\%), the patient is too ill to address sexual health (54\%), sexuality is not seen as a problem by the patient $(53 \%)$, and lack of organizational policy (49\%). Nurses who rarely or never address sexual health mentioned the following barriers: lack of organizational policy (79\%), lack of training $(80 \%)$, the patient did not initiate the subject $(72 \%)$, lack of knowledge on how to initiate the subject $(71 \%)$, the older age of the patient $(60 \%)$, and lack of knowledge on the topic in general (57\%).

Barriers that differed significantly between nurses who do and who do not discuss sexual health with their patients were: lack of knowledge (57\% vs. $23 \%$, $p=0.001$ ), lack of training ( $80 \%$ vs. $43 \%, p<0.001$ ), not knowing how to initiate the subject $(71 \%$ vs. $32 \%, p=0.001)$, "I would find it unpleasant myself" ( $18 \%$ vs. $2 \%$, $p=0.015)$, "It makes me feel uncomfortable" $(20 \%$ vs. $5 \%, p=0.036)$, the patient does not initiate the subject $(72 \%$ vs. $24 \%, p<0.001)$, and lack of organizational policy (79\% vs. $49 \%, p<0.001$ ) (Figure 4$)$.

\section{Discussion}

This is the first study on HF nurses that explores the preferred moment of discussing sexual health and providing sexual counseling, and that examines differences in barriers between those nurses who do and those who do not assess sexual health with their patients. 
Our findings suggest that the majority of HF nurses (61\%) does not assess sexual health, although the HF nurses (74\%) in our study do feel responsible for discussing sexual health with their patients and offering them sexual counseling. This finding is comparable with other studies that looked at the feeling of responsibility experienced by cardiac healthcare providers and the practice regarding sexual counseling in cardiac settings. In a European survey on the practice and responsibility of nurses to provide sexual counseling to cardiac patients, $87 \%$ of the cardiac nurses agreed that nurses have a responsibility to discuss sexual health. However, only $11 \%$ of the nurses discussed the subject frequently with their patients. ${ }^{10}$ Furthermore, a US study on nurses working with patients after a myocardial infarction and national surveys in Ireland and Sweden in cardiac rehabilitation centers and coronary care unit teams have shown comparable findings. ${ }^{11,14,16}$ It can be concluded that not discussing sexual health and providing sexual counseling in different cardiac settings is a general problem that does not only relates to HF nurses, but also other cardiac healthcare providers. This limited implementation of sexual counseling in practice may rightfully be called a concern, because a considerable number of HF patients experience sexual difficulties which have a negative effect on their own quality of life and that of their partners. ${ }^{6,17}$ Patients worry about resuming sexual activity and are in need of education and counseling on the topic of sexual health. ${ }^{1,2}$ Furthermore, previous research has shown that patients also experience barriers to initiating this topic. ${ }^{7}$

Therefore, to improve treatment of cardiac patients, more attention needs to be given to sexual counseling in nursing education. However, in order to educate nurses successfully on this subject, it is important to know which barriers keep nurses from assessing sexual health and providing counseling. We found that the most 
frequently reported barrier to addressing sexual concerns was the lack of an organizational policy. This is a surprising find, because HF guidelines recommend that healthcare providers assess sexual health with both male and female patients and their partners. ${ }^{8,9}$ The current class of recommendation given to this subject is I, which indicates that counseling on sexual activity is considered to be very important. However, the results from our study suggest that HF clinics in the Netherlands do not employ a policy that ensures that patients are structurally educated and counseled on this subject. To improve the discussion of sexual health with patients, a first step could be to implement these guidelines in treatment protocols.

We found subtle differences in barriers perceived by nurses who do and nurses who do not discuss sexual health with their patients. Nurses who do discuss sexual health experience more barriers related to patient factors, such as issues related to culture, religion, language, ethnicity, the severity of the illness, and the nurses' belief that the patient does not see sexuality as a problem. On the other hand, nurses who do not discuss sexual health experience more barriers related to external factors, such as lack of training, lack of general knowledge, lack of knowledge on how to initiate the topic. and the fact that the patient does not initiate the subject. Additionally, nurses who do discuss sexual health with their patients more often reported to have gone on to higher education regarding sexual counseling. When considering the abovementioned differences in barriers, to improve treatment nurses might benefit from specific training courses to help them gain more knowledge about sexual health, to acquire communication skills, and to feel more comfortable discussing this topic.

Furthermore, it might help nurses to discuss sexual health at a moment which is most comfortable for them. According to our findings this is during a follow-up visit 
at the HF clinic or when medication is discussed. This result confirms our finding that nurses working at HF clinics believe they are responsible for assessing sexuality; they do not delegate this task to their colleagues working at the rehabilitation centre. Nurses could use validated questionnaires to initiate the topic of sexual health, or they could try to address the topic while discussing medication with the patient. ${ }^{18}$ The moment when medication (e.g., beta-blockers) is up-titrated provides the perfect opportunity for nurses to enquire after changes in sexual responses or fear of impotence. Nurses could also benefit from a practical workshop in which they can practice the approach that would suit them best. Furthermore, it can be helpful if such programs look at the roles the different team members play in assessing sexuality and providing sexual counseling to patients. Taking these educational courses can be advantageous, because the improved knowledge and attitudes of nurses will help them feel at ease when discussing sexual health in practice. ${ }^{19}$ Steinke et al. have suggested a number of strategies that can be used in patient education and management. ${ }^{20}$ For example, they suggest to initiate the topic of sexual health after discussing exercise recommendations. To this end, they recommend employing a general statement, such as "Many individuals with HF have questions and concerns about resuming sexual activity. What concerns do you have?" In addition, Mick wrote an article which provides 10 strategies to help nurses who work with oncology patients address and validate patients' sexual experiences and concerns about quality of life. ${ }^{21}$ Such strategies could easily be translated to cardiology practice. Mick's strategies for nurses to understand sexuality include: providing information, addressing causes of discomfort, and being an objective listener. ${ }^{21}$

A limitation of this study is the generalizability of the results. We are aware of the fact that our study sample might be too homogenous. For instance, we have sent 
three copies of the questionnaire to all HF clinics in the Netherlands, but did not direct the questionnaires to the nurses personally. We expected the response to be higher if the questionnaires were addressed anonymously. However, a consequence of this approach is that we only acquired information on the demographics of nurses who did respond, and therefore lack information on nurses who did not respond. Furthermore, it is reasonable to expect that mostly nurses who already have an affinity with the subject completed and returned the questionnaire. This could have resulted in an overrepresentation of the percentage of nurses who not only feel responsible for addressing the topic of sexual health, but also put this in practice. It is imaginable that nurses who do not recognize the importance of the subject, and therefore do not feel responsible for discussing sexual health with their patients, also did not complete the questionnaire. A second limitation of our study is that we did not perform psychometric testing on the complete questionnaire; we only tested for facevalidity. Although the questionnaires have been used before in a European population, they were not primary developed for the Dutch population. ${ }^{8}$

It can be concluded that although nurses feel they have a responsibility to discuss their patients' sexual health, sexuality is rarely discussed in HF clinics. The preferred moment of initiating this topic is during a follow-up visit to the outpatient clinic or when medication is discussed. Barriers experienced by nurses to discussing sexuality are related to personal, patient and organizational factors.

To improve patient care, interventions and training courses should focus on improving both nurses' knowledge about sexual health and their communication skills. Such educational courses should be practical and aimed at making nurses feel at ease discussing matters of sexuality in daily practice. The roles different team members could play and the possibilities for referral to other professions also need to 
be taken into account. Furthermore, the HF guidelines should be employed to include sexual counseling in the policy of organizations.

\section{Acknowledgements}

We are grateful to the HF nurses who took the time to participate in this research. We would like to thank the steering committee members for their help: R.Y. Schouten, J.M.A. Visser-Meily, P. Ninaber, J.E. de Groot, C.C. Tuijnman-Raasveld, K. Kanselaar, P. Rabsztyn and W.J. Louridtz. 


\section{References}

1. Jaarsma T. Sexual problems in heart failure patients. Eur J Cardiovasc Nurs. 2002;1:61-7.

2. Jaarsma T, Dracup K, Walden J, Stevenson LW. Sexual function in patients with advanced heart failure. Heart Lung. 1996;25:262-70.

3. Mandras SA, Uber PA, Mehra MR. Sexual activity in chronic heart failure. Mayo Clin Proc. 2007;82:1203-10.

4. Rosen RC, Kostis JB, Jekelis A, Taska LS. Sexual sequelae of antihypertensive drugs: treatment effects on self-report and physiological measures in middleaged male hypertensives. Arch Sex Behav 1994;23:135-52.

5. Silvestri A, Galetta P, Cerquetani E, Marazzi G, Patrizi R, Fini M, Rosano GM. Report of erectile dysfunction after therapy with beta-blockers is related to patient knowledge of side effects and is reversed by placebo. Eur Heart $\mathrm{J}$ 2003;24:1928-32.

6. Westlake C, Dracup K, Walden JA, Fonarow G. Sexuality of patients with advanced heart failure and their spouses or partners. J Heart Lung Transplant. 1999;18:1133-8.

7. Gott M. Hinchliff S. Barriers to seeking treatment for sexual problems in primary care: a qualitative study with older people. Fam Pract. 2003;20:690-5.

8. Dickstein K, Cohen-Solal A, Filippatos G, McMurray JJ, Ponikowski P, PooleWilson PA et al. ESC guidelines for the diagnosis and treatment of acute and chronic heart failure 2008. Eur J Heart Fail. 2008;10:933-89.

9. Heart Failure Society Of America. HFSA 2006 Comprehensive Heart Failure Practice Guideline. J Card Fail. 2006;12:10-38. 
10. Jaarsma $\mathrm{T}$, Strömberg $\mathrm{A}$, Fridlund $\mathrm{B}$, De Geest $\mathrm{S}$, Mårtensson J, Moons $\mathrm{P}$ et al. Sexual counselling of cardiac patients: nurses' perception of practice, responsibility and confidence. Eur J Cardiovasc Nurs. 2010;9:24-9.

11. Doherty S, Byrne M, Murphy AW, McGee HM. Cardiac rehabilitation staff views about discussing sexual issues with coronary heart disease patients: a national survey in Ireland. Eur J Cardiovasc Nurs. 2011;10:101-7.

12. Jaarsma T, Tan B, Bos RJ, van Veldhuisen DJ. Heart failure clinics in the Netherlands in 2003. Eur J Cardiovasc Nurs. 2004;3:271-4

13. Jaarsma T, Haaijer-Ruskamp FM, Sturm H, Van Veldhuisen DJ. Management of heart failure in The Netherlands. Eur J Heart Fail. 2005;7:371-5.

14. Steinke EE, Patterson-Midgley P. Sexual counselling of MI patients: nurses' comfort, responsibility, and practice. Dimensions of Critical Care Nursing. 1996;15:216-23.

15. Waterhouse JK. Development and testing of a structural equation model of nursing practice related to sexuality. Doctoral dissertation. University of Delaware, Newark, DE; 1996.

16. Ivarsson B, Fridlund B, Sjöberg T. Information from health care professionals about sexual function and coexistence after myocardial infarction: a national survey. Heart Lung. 2009;38:3350-5.

17. Hoekstra T, Jaarsma T, Sanderman R, van Veldhuisen DJ, Lesman-Leegte I. Perceived sexual difficulties and associated factors in patients with heart failure. Am Heart J. 2012;163:246-51.

18. Jaarsma T, Steinke EE, Gianotten WL. Sexual problems in cardiac patients: how to assess, when to refer. J Cardiovasc Nurs. 2010;25:159-64. 
19. Post MWM, Gianotten WL, Heijnen L, Lambers EJHR, Willems M. Sexological competence of different rehabilitation disciplines and effects of a disciplinespecific sexological training. Sex Disabil. 2008;26:3-14.

20. Steinke EE, Mosack V, Wright DW, Chung ML, Moser DK. Risk factors as predictors of sexual activity in heart failure. Dimens Crit Care Nurs. 2009;28:123-9.

21. Mick JM. Sexuality assessment: 10 Strategies for improvement. Clin J Oncol Nurs. 2007;11:671-5. 


\section{Figure titles and legends}

Figure 1. Practice of discussing sexual health by HF nurses

Figure 2. Perceived responsibility of HF nurses to discuss sexual health

Figure 3. Preferred moment of discussing sexual health 
Table 1. Nurse characteristics

\begin{tabular}{|c|c|c|c|c|}
\hline & $\begin{array}{l}\text { Total } \\
(n=146)\end{array}$ & $\begin{array}{l}\text { Nurses who } \\
\text { discuss sexual } \\
\text { health }(n=58)\end{array}$ & $\begin{array}{l}\text { Nurses who do not } \\
\text { discuss sexual } \\
\text { health }(\mathrm{n}=88)\end{array}$ & $p$ \\
\hline Female gender & $83 \%$ & $81 \%$ & $88 \%$ & .236 \\
\hline Age & $45 \pm 8$ & $45 \pm 8$ & $46 \pm 7$ & .830 \\
\hline \multicolumn{5}{|l|}{ Marital status } \\
\hline Married/living with a partner & $88 \%$ & $88 \%$ & $87 \%$ & \\
\hline Never married & $9 \%$ & $12 \%$ & $7 \%$ & .353 \\
\hline Divorced/widowed & $3 \%$ & $0 \%$ & $6 \%$ & \\
\hline \multicolumn{5}{|l|}{ Years in cardiac nursing } \\
\hline $0-5$ years & $32 \%$ & $31 \%$ & $32 \%$ & \\
\hline $6-10$ years & $32 \%$ & $38 \%$ & $28 \%$ & .422 \\
\hline$>10$ years & $36 \%$ & $31 \%$ & $40 \%$ & \\
\hline \multicolumn{5}{|l|}{ Highest level of education } \\
\hline Basic nursing training & $14 \%$ & $10 \%$ & $17 \%$ & \\
\hline Bachelor degree & $67 \%$ & $69 \%$ & $67 \%$ & .654 \\
\hline Master degree & $5 \%$ & $5 \%$ & $5 \%$ & \\
\hline Other & $14 \%$ & $16 \%$ & $11 \%$ & \\
\hline Education in sexual counseling & $30 \%$ & $41 \%$ & $22 \%$ & .012 \\
\hline
\end{tabular}


Table 2. Barriers to addressing sexual concerns of nurses who do and nurses who do not discuss sexual health with their patients

\begin{tabular}{|c|c|c|c|c|}
\hline Barrier & $\begin{array}{l}\text { Total } \\
\text { group } \\
(n=146)\end{array}$ & $\begin{array}{c}\text { Nurses who do } \\
\text { discuss sexuality } \\
\qquad(n=58)\end{array}$ & $\begin{array}{c}\text { Nurses who do } \\
\text { not discuss } \\
\text { sexuality ( } n=88 \text { ) }\end{array}$ & p-value \\
\hline $\begin{array}{l}\text { Lack of organizational } \\
\text { policy }\end{array}$ & $67 \%$ & $49 \%$ & $79 \%$ & $<.001$ \\
\hline Lack of training & $42 \%$ & $43 \%$ & $80 \%$ & $<.001$ \\
\hline $\begin{array}{l}\text { Issues relating to } \\
\text { culture and religion }\end{array}$ & $40 \%$ & $68 \%$ & $53 \%$ & .121 \\
\hline Older aged patient & $39 \%$ & $43 \%$ & $60 \%$ & .084 \\
\hline $\begin{array}{l}\text { Issues relating to } \\
\text { language and ethnicity }\end{array}$ & $38 \%$ & $56 \%$ & $54 \%$ & .832 \\
\hline $\begin{array}{l}\text { Not knowing how to } \\
\text { initiate the subject }\end{array}$ & $35 \%$ & $32 \%$ & $71 \%$ & $<.001$ \\
\hline $\begin{array}{l}\text { Patient does not initiate } \\
\text { the subject }\end{array}$ & $35 \%$ & $24 \%$ & $72 \%$ & $<.001$ \\
\hline $\begin{array}{l}\text { Patient is too ill to } \\
\text { address sexual issues }\end{array}$ & $35 \%$ & $54 \%$ & $45 \%$ & .395 \\
\hline Lack of knowledge & $28 \%$ & $23 \%$ & $57 \%$ & .001 \\
\hline $\begin{array}{l}\text { Sexuality is not seen as } \\
\text { a problem for the } \\
\text { patient }\end{array}$ & $22 \%$ & $53 \%$ & $33 \%$ & .074 \\
\hline $\begin{array}{l}\text { Sexuality is a private } \\
\text { matter }\end{array}$ & $19 \%$ & $23 \%$ & $33 \%$ & .253 \\
\hline
\end{tabular}




\begin{tabular}{|c|c|c|c|c|}
\hline $\begin{array}{l}\text { Concerns about } \\
\text { increasing patients' } \\
\text { anxiety and discomfort }\end{array}$ & $19 \%$ & $17 \%$ & $33 \%$ & .078 \\
\hline $\begin{array}{l}\text { Patients' lack of } \\
\text { readiness }\end{array}$ & $19 \%$ & $45 \%$ & $32 \%$ & .225 \\
\hline $\begin{array}{l}\text { Presence of a third } \\
\text { party }\end{array}$ & $18 \%$ & $23 \%$ & $24 \%$ & .906 \\
\hline Not enough time & $13 \%$ & $19 \%$ & $12 \%$ & .275 \\
\hline Embarrassment & $10 \%$ & $14 \%$ & $16 \%$ & .837 \\
\hline $\begin{array}{l}\text { Feeling no connection } \\
\text { with the patient }\end{array}$ & $10 \%$ & $11 \%$ & $15 \%$ & .561 \\
\hline $\begin{array}{l}\text { It makes me } \\
\text { uncomfortable }\end{array}$ & $9 \%$ & $5 \%$ & $20 \%$ & .036 \\
\hline $\begin{array}{l}\text { I would find it } \\
\text { unpleasant myself }\end{array}$ & $8 \%$ & $2 \%$ & $18 \%$ & .015 \\
\hline $\begin{array}{l}\text { Fear of offending the } \\
\text { patient }\end{array}$ & $8 \%$ & $11 \%$ & $12 \%$ & .979 \\
\hline $\begin{array}{l}\text { Too large an age } \\
\text { difference between you } \\
\text { and the patient }\end{array}$ & $6 \%$ & $2 \%$ & $11 \%$ & .071 \\
\hline Someone else's job & $3 \%$ & $0 \%$ & $6 \%$ & .075 \\
\hline $\begin{array}{l}\text { Patient is of the } \\
\text { opposite sex }\end{array}$ & $1 \%$ & $0 \%$ & $1 \%$ & .400 \\
\hline $\begin{array}{l}\text { Patient is of the same } \\
\text { sex }\end{array}$ & $0 \%$ & $0 \%$ & $0 \%$ & - \\
\hline
\end{tabular}


